\title{
A NOVEL ALGORITHM FOR PREDICTING RANKS BASED ON TEXTUAL REVIEWS
}

\#1 M.Tech Scholar, Department of Computer Science and System Engineering, Andhra University College of Engineering (A), Visakhapatnam, AP, India.

\#2 Professor, Department of Computer Science and System Engineering, Andhra University College of Engineering (A), Visakhapatnam, AP, India.

\section{ABSTRACT}

Data Mining is described as the computational process of discovering patterns in large data involving methods at the intersection of artificial intelligence, ML approach, statistics, and database systems. As the main goal of mining is to extract the valuable information from large data sets, it should also concentrate on the privacy attributes that are related to sensitive data extraction. Now a day's, we have come with a wide range of review websites that presents a good opportunity to share our view points about the products what we purchase. But there is no accurate website which can provide the exact rating from the text reviews. As we know that almost all the review websites are facing with overloading problem with continuous duplicate reviews that were posted by the same user. The main limitation with current review websites is how to mine valuable information from reviews to understand a user's preferences and make an accurate recommendation is crucial part. Primitive recommendation systems (RS) concentrate mainly with some factors, like users purchase records, category of products that are available in the site as well the purchase location. In this current thesis, we mainly propose a sentiment-based rating prediction method (RPS) to improve prediction accuracy in recommender systems. Initially we developed a social user sentimental measurement approach and calculate each user's sentiment on items/products. By conducting various experiments on our proposed model by taking a local web interface by taking some products into assumption, our simulation results show the sentiment can well characterize user preferences, which help to improve the recommendation performance.

Key Words:

Sentiment Analysis, Privacy, Database Systems, Rating Prediction Systems, Data Mining,

\section{INTRODUCTION}

Data mining is the process of extracting useful or structured information from a raw or un-structured data[1]. Generally this is used mainly in performing operations like insurance sector, bank and retail sector, hospital for identifying diseases, shopping malls to calculate the priority of items that were sold. Data mining is the evaluation connected with info regarding interactions who have not necessarily previously recently been found [2]. One example is, the 
actual gross sales documents for the distinct model of tennis racket may possibly, in case adequately reviewed as well as linked to different industry info, show the temporary [3]. It is very challenging to comprehend or perhaps further evaluation of that massive data bank. Between the many styles we are meant to discover the many recurrent styles. The best way to query recurrent styles: the style that develops generally in a dataset is really a recurrent style; we've many algorithms to query recurrent styles just like apriori criteria, FP- increase criteria.

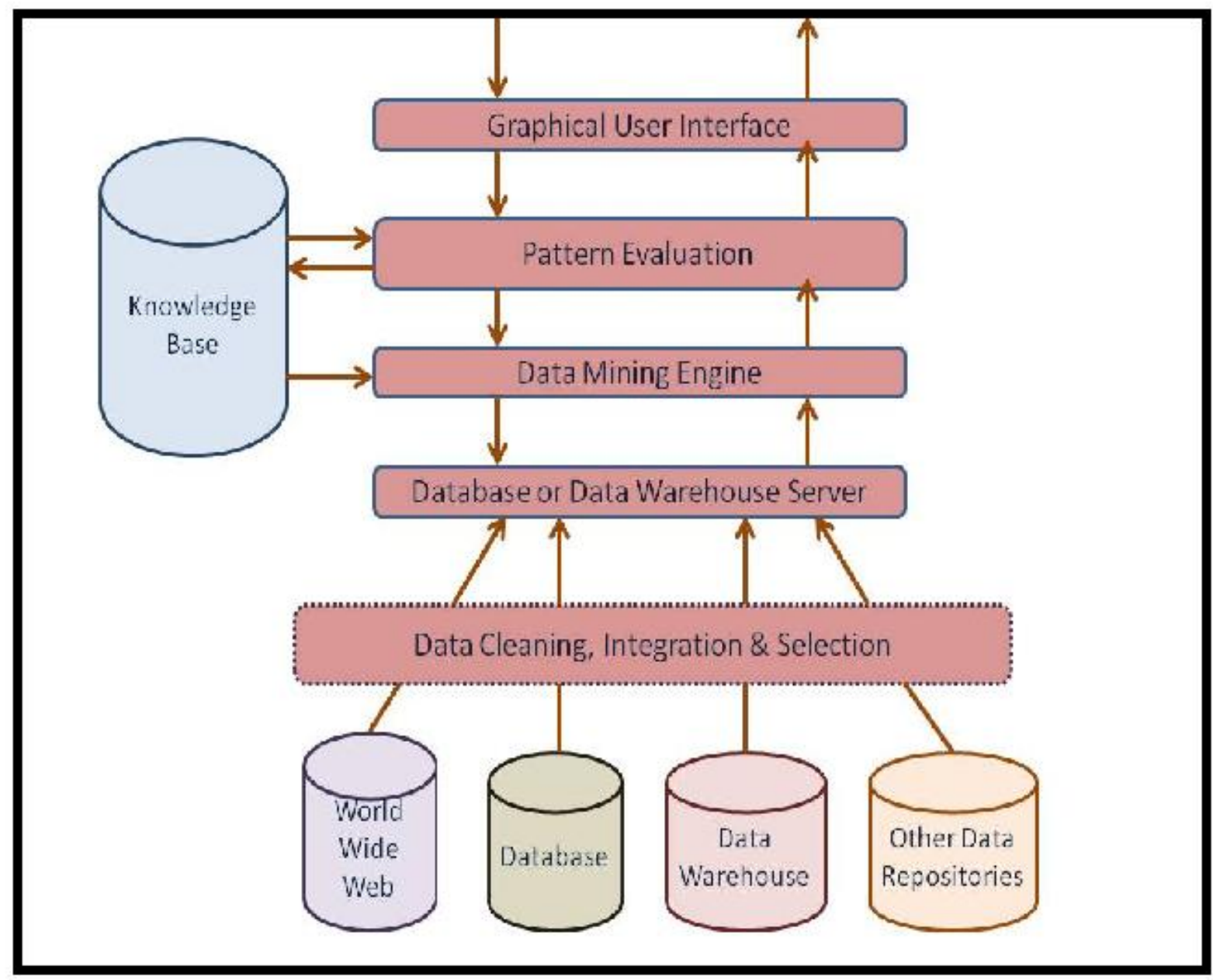

\section{FIGURE. 1. REPRESENTS THE BASIC ARCHITECTURE OF A DATA MINING AS A CORE METHOD IN THE PROCESS OF KNOWLEDGE DISCOVERY}

From the above figure 1, we can clearly find out that mining engine is one of the part or one core step in the process of knowledge discovery. Initially the data set will be collected from either world wide web or data base or from other data repositories [4], [5] and cleaned in order to reduce the redundant items or irrelevant items that are available in the data sets, once the data set is cleaned and pre-processed then we can able to send for the next process like classification and in turn for clustering and rule mining by using various data mining algorithms. One among the several algorithms in classification is Fp-miner where this is used for clustering the frequent item sets from a set of distinct elements. Once the data mining algorithms are done then it will be send 
to the pattern evaluation phase, where the data will be matched with the knowledge base and once the data is verified then it will be visualized as result.Despite the fact that there is a great deal of individual data that is accessible in online textual reviews, this data assumes an extremely key part on basic decision making. For instance, in the event that we need to consider the best case for the textual reviews, the user will choose what to purchase on the off chance that he or she sees valuable reviews posted by others, particularly by the client's companions or companion of companions. Presently a days we can likewise see the rating stars alongside content based surveys under the items that are accessible on the web. The user will choose the item is great in the event that it contains the most worthy rating stars alongside number of positive reviews are more, a similar client feel that reviews is awful if the most number of text are negative and less number of stars given for that suitable item. We believe reviews and reviewers will do help to the rating prediction based on the idea that high-star ratings may greatly be attached with good reviews . Consequently, how to extract useful information review and the connection between analysts in informal communities has turned into a vital issue in web mining, machine learning and characteristic dialect preparing.

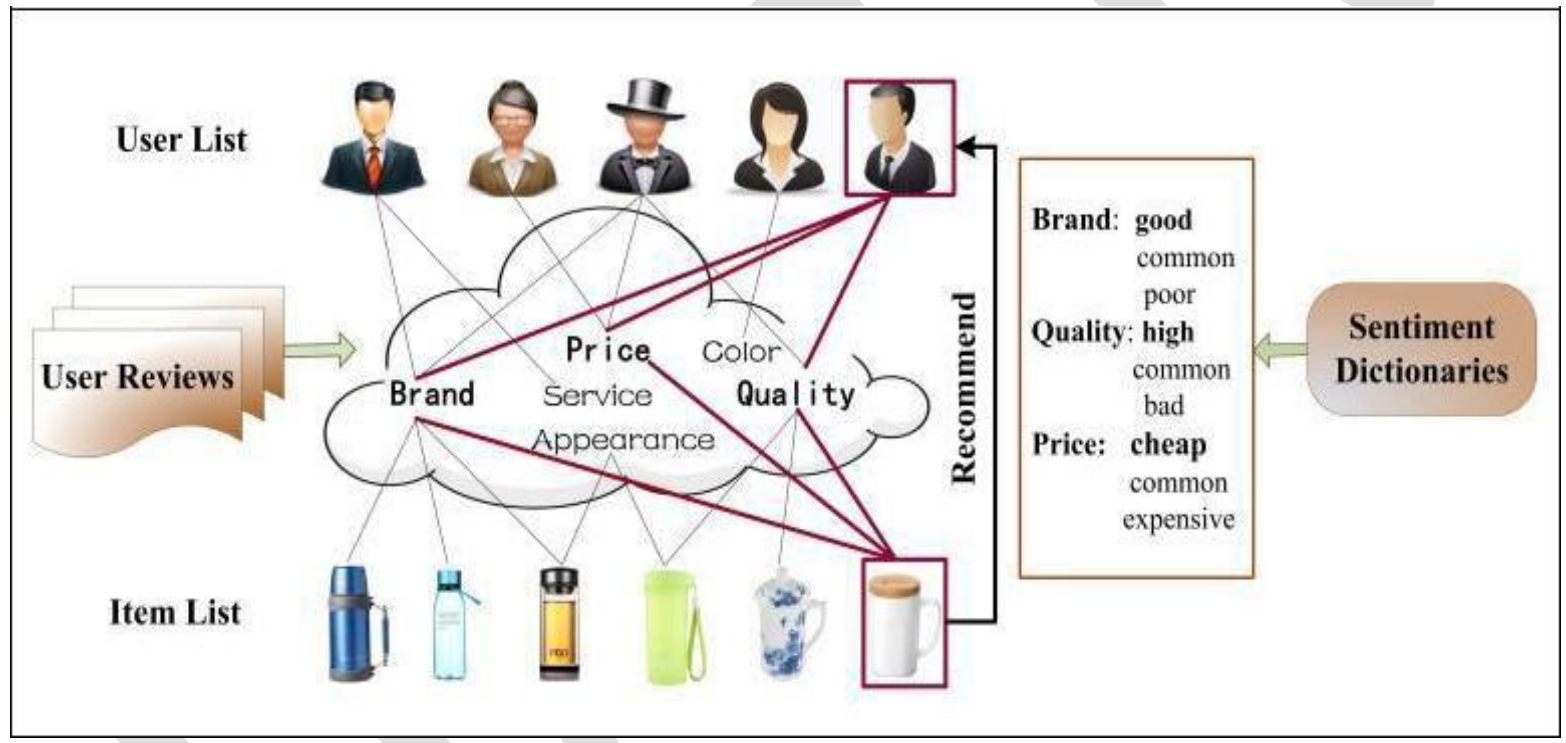

FIGURE. 2. REPRESENTS THE WORKING FLOW OF SENTIMENT ANALYSIS FOR EXTRACTING THE VALUABLE INFORMATION FROM THE PRODUCT REVIEWS

In addition to extracting user preferences, we must also consider interpersonal views. Numerous methodologies about the interpersonal influence social networks have proved good performance in recommendation, which can successfully resolve the "cold start" issues. However, the current approaches mainly leverage product category information or tag information to study the interpersonal influence. These strategies are all limited on the structured data, which isn't generally accessible on a few sites. However, user reviews can give us thoughts in interpersonal inference and user preferences. To address these issues, we propose a supposition based rating forecast technique in the system of matrix factorization. In our work, we make use of social user's sentiment to infer ratings. 
Figure. 2 is an example that illustrates our motivation. First, we extract product features from user reviews. Then, we find out the sentiment words, which are used to describe the product features. Besides, we leverage sentiment dictionaries to calculate sentiment of a specific user on an item/product. What is more, we combine social friend circle with sentiment to recommend. In Figure.2, the last user is interested in those product features, so based on the user reviews and the sentiment dictionaries, the last item will be recommended. Compared with previous work [8], [9], the main difference is that: we use unstructured information to recommend instead of other structured social factors. Compared with [11]-[15], the main difference is that: their work mainly focuses on classifying users into binary sentiment (i.e. positive or negative), and they do not go further in mining user's sentiment. In our paper, we not only mine social user's sentiment, but also explore interpersonal sentimental influence and item's reputation. Finally, we take all of them into the recommender system.

The main contributions of our approach are as follows: 1) we propose a user sentimental measurement approach, which is based on the mined sentiment words and sentiment degree words from user reviews. Besides, some scalable applications are proposed. For example, we explore how the mined sentiment spread among user's friends. What is more, we leverage social user's sentiment to infer item's reputation, which showed great improvement in accuracy of rating prediction. 2) We make use of sentiment for rating prediction. User sentiment similarity focuses on the user interest preferences. User sentiment influence reflects how the sentiment spreads among the trusted users. 3) Item reputation similarity shows the potential relevance of items. We fuse the three factors: user sentiment similarity, interpersonal sentimental influence, and item reputation similarity into a probabilistic matrix factorization framework to carry out an accurate recommendation. The experimental results and discussions show that user's social sentiment that we mined is a key factor in improving rating prediction performances.

\section{RELATED WORK}

In this section we mainly discuss about the related work that was carried out in finding the rating prediction based on the social sentiment from textual reviews. In this section we mainly discuss about the basic data mining algorithms that are classified and various data mining models. Now let us discuss about this in detail

\section{PRILEMINARY KNOWLEDGE}

Data mining processes have required an integration of techniques from multiple disciplines such as statistics, machine learning, database technology, pattern recognition, neural networks, information retrieval and spatial data analysis. The process of data mining involves a keen observation with a set of algorithms to accomplish different tasks. All these algorithms attempt to fit a model to the given data set. All the data mining algorithms are mainly used to examine the input data and then determine a model that is very closest to the characteristics of the data being examined.

Generally the data mining algorithms can be classified into different ways based on the following three things like:

1. Model Based: The purpose of the algorithm is to fit a model to the required data. 
2. Preference Based: This is based on the preferences or criteria that are being used.

3. Search Based: In this method, we mainly find out the search techniques that are being used.

\section{THE PROPOSED RATING PREDICTION METHOD (RPS) USING USER TEXTUAL SENTIMENTAL APPROACH}

In this section we mainly discuss about the proposed RPS using user textual sentimental approach. Now let us discuss about this proposed RPS method in detail as follows:

\section{MOTIVATION}

The main aim or preliminary knowledge that was carried out in order to propose the current RPS approach is to find the effective clues from text based reviews and try to predict social user's ratings. In this paper, we mainly extract product features from user review corpus, and then we introduce the method of identifying social user's sentiment. In addition, we describe the three sentimental factors. At last we fuse all of them into our sentiment-based rating prediction method (RPS). The following sub-sections describe more details about our approach.

\section{METHOD OF EXTRACTING THE PRODUCT FEATURES}

Initially we mainly concentrate on that extraction of product features which mainly describe about the issues that are related to the products. In this thesis, we extract product features from textual reviews using LDA [11]. We mainly want to get the product features including some named entities and some product/item/service attributes. LDA is a Bayesian model, which is utilized to model the relationship of reviews, topics and words. In Figure. 3, the shaded variables indicate the observed variables and the unshaded variables indicate the latent variables. The arrow indicates a conditional dependency between the variables and plates represented by the box. The definition of terminologies in LDA model is described as:

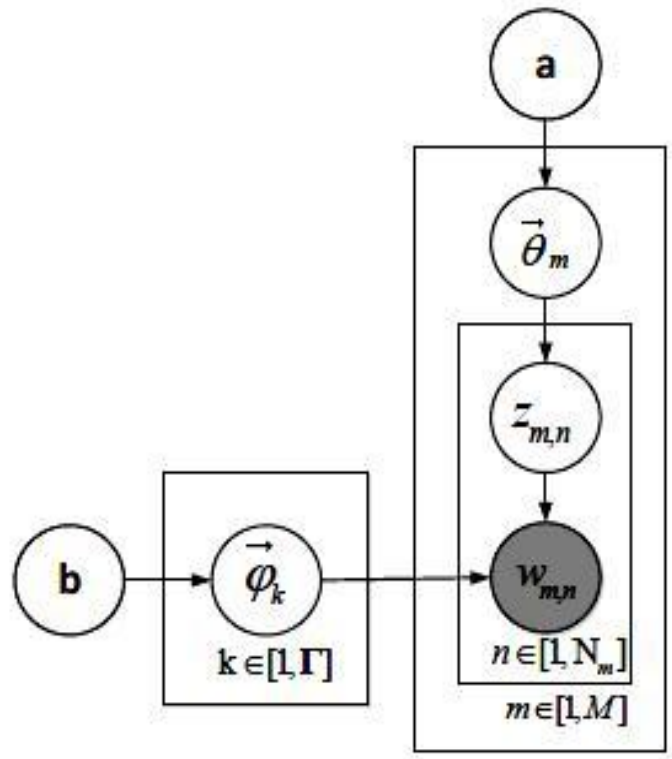

FIGURE. 3. REPRESENTS THE PICTORAIL NOTATION OF LDA 
If we look at the figure 3 , the borders that are available mainly represents the replicates and the outer border clearly represents the user documents and the inner border that is available represent the repeated choice of words and topics that are available within the document.

V: the vocabulary, it has $N d$ unique words. Each word is presented by the corresponding label $\{1,2, \cdots$, $N d\}$.

$\boldsymbol{\nabla} \boldsymbol{i} \in\{1,2, \cdots, N d\}$ : the word, each word of a review is mapped to $\boldsymbol{V}$ whose size is $N d$ through character matching.

$>\boldsymbol{d} m$ : the document/review of a user, it corresponds to a word set of the review. A user with only one document. All documents denote as $D=\{d 1, d 2, \cdots, d M\}$.

$>\Gamma$ : the number of topics (const scalar).

$>\boldsymbol{\theta}^{\rightarrow} \mathbf{m}$ : the multinomial distribution of topics specific to the document $m$. One proportion for each document, $\Theta=\{\theta m\} m=1 M(M \times \Gamma$ matrix $)$

$>\vec{\varphi} \vec{k}$ : the component for each topic, $\Phi=\{\vec{\varphi} k\} k=1 \Gamma(\Gamma \times k$ matrix $)$

$\boldsymbol{z} \boldsymbol{m}, \boldsymbol{n}$ : the topic associated with the $n$-th token in the document $m$.

$>\boldsymbol{a}, \boldsymbol{b}$ : Dirrichlet priors to the multinomial distribution $\theta m$ and $\vec{\varphi} k$.

\section{DATA PREPROCESSING FOR LDA}

To construct the vocabulary, we firstly regard each user's review as a collection of words without considering the order. Then we filter out "Stop Words" [34, 41], "Noise Words" and sentiment words, sentiment degree words, and negation words. A stop word can be identified as a word that has the same likelihood of occurring in those documents not relevant to a query as in those documents relevant to the query. For example, the "Stop Words" could be some prepositions, articles, and pronouns etc.. After words filtering, the input text is clear and without much interference for generating topics. All the unique words are constructed in the vocabulary $V$, each word has a label $w i \in\{1,2, \cdots, N d\}$.

\section{THE GENERATIVE PROCESS OF LDA}

The input of LDA model is all users' document sets $D$, and we assign the number of topic $\Gamma$ (we set 50 empirically). The output is the topic preference distribution for each user and a topic list, which contains at least 10 feature words under each topic. The generative process of LDA consists of three steps:

$>$ For each document $\boldsymbol{d} j$, we choose a dimensional Dirichlet random variable $\theta m \sim$ Dirichlet (a).

$>$ For each topic $z k$, where $k \epsilon[1, \Gamma]$, we choose $\phi k \sim$ Dirichlet (b). For each topic $z k$, the inference scheme is based upon the observation that:

$p(\Theta, \Phi \mid$ Dtrain , $\alpha, b)=\Sigma p(\Theta, \Phi \mid z$, Dtrain , $\alpha, b) z P(z, \mid$ Dtrain , $\alpha, b)(3)$.We obtain an approximate posterior on $\Theta$ and $\Phi$ by using a Gibbs sampler to compute the sum over $z$.

$>$ Repeating the process above and eventually we get the output of LDA. 


\section{USER SENTIMENTAL MEASUREMENT}

We extend HowNet Sentiment Dictionary3 [12] to calculate social user's sentiment on items. In our paper, we merge the positive sentiment words list and positive evaluation words list of HowNet Sentiment Dictionary into one list, and named it as POS-Words; also, we merge the negative sentiment words list and negative evaluation words list of HowNet Sentiment Dictionary into one list, and named it as NEG-Words. Our sentiment dictionary (SD) includes 4379 POS-Words and 4605 NEG-Words. Besides, we have five different levels in sentiment degree dictionary (SDD), which has 128 words in total. There are 52 words in the Level-1, which means the highest degree of sentiment, such as the words "most", and "best". And 48 words in the Level-2, which means higher degree of sentiment, such as the words "better", and "very". There are 12 words in the Level-3, such as the words "more", and "such". There are 9 words in the Level-4, such as the words "a little", "a bit", and "more or less". And there are 7 words in the Level-5, such as the words "less", "bit", and "not very". Also, we built the negation dictionary (ND) by collecting frequently-used negative prefix words, such as "no", "hardly", "never", etc. These words are used to reverse the polarity of sentiment words. The representative words and the sizes of all dictionaries are introduced in Table 1.

\section{TABLE 1.: BRIEF INTRODUCTION OF THE SENTIMENT DICTIONARIES}

\begin{tabular}{|c|l|}
\hline Dictionaries & \multicolumn{1}{|c|}{ REPRESENTATIVE WORDS } \\
\hline \multirow{3}{*}{ SD(8938) } & $\begin{array}{l}\text { POS-Words(4379):attractive, clean, beautiful, comfy, } \\
\text { convenient, delicious, delicate, exciting, fresh, happy, } \\
\text { homelike, nice, ok, yum ... } \\
\text { NEG-Words(4605):annoyed, awful, bad, poor, } \\
\text { boring, complain, crowed, dirty, expensive, hostile, } \\
\text { sucks, terribly, unfortunate, worse ... }\end{array}$ \\
\hline \multirow{3}{*}{ ND(56) } & $\begin{array}{l}\text { no, nor, not, never, nobody, nothing, none, neither, } \\
\text { few, seldom, hardly, haven't, can't, couldn't, don't, } \\
\text { didn't, doesn't, isn't, won't,... }\end{array}$ \\
\hline \multirow{3}{*}{ SDD(128) } & $\begin{array}{l}\text { Level-1 (52): most, best, greatest, absolutely, } \\
\text { extremely, highly, excessively, completely, entirely, } \\
\text { 100\%, highest, sharply, superb... } \\
\text { Level-2 (48): awfully, better, lot, very, much, over, } \\
\text { greatly, super, pretty, unusual... } \\
\text { Level-3 (12): even, more, far, so, further, intensely, } \\
\text { rather, relatively, slightly more, insanely, comparative. } \\
\text { Level-4 (9): a little, a bit, slight, slightly, more or less, } \\
\text { relative, some, some what, just. } \\
\text { Level-5 (7): less, not very, bit, little, merely, passably, } \\
\text { insufficiently. }\end{array}$ \\
\hline
\end{tabular}

\section{BENCH MARK FOR THE PRODUCTS AS AN EXTENSION}

The term bench-mark, or benchmark, actually came from the Chinese horizontal marks that was made in stone structures by various surveyors, into which an angle-iron could be placed to form a "bench" for a leveling rod, thus ensuring that a leveling rod could be accurately repositioned in the same place in the future. This term is generally given for any item to mark a point as an elevation reference. Actually if we look in online shopping there are several items that come under one category and where the customer or user is unable to decide which item is best and which item is worst from a set of items. So if there is any option like bench mark for 
classifying the items, then it is a great advantageous for the end users to easily identify the best product from a set of products which are available in the database. Normally the bench mark will be indicated or measured between 0 to 5 or for some web sites they may mark from 1 to 5.Actually for every product if the product is not having any review or feedback , at that stage the bench mark will be like 0.0.If the same product has equal number of positive and equal number of negative reviews or feedback, then it will be measured between 2.5 to 3.0.If the same product is having only positive reviews other than negative from overall ratings, then it will be almost like 5.0. So based on the value that was allotted for the product the customer can able to figure out easily which product is having the good feedback and positive reviews and which one is having negative impact. So if this type of facility is available for every online shopping sites, it is a great benefit for the customers to avoid the fraud to be occurred during online shopping or online prediction.

In our proposed application we try to use the same Bench mark concept to the categorize the products based on the text based reviews and based on the positive or negative or neutral keywords. Here in our proposed application we mainly calculate the bench mark based on three types of feedbacks like Positive, Negative and Neutral. By considering all the three constrains we are going to implement the proposed bench mark concept in our application.

\section{PSEUDO CODE FOR BENCH MARK CALCULATION}

String Bench;

//Here we assumed $\mathrm{K}$ as the Overall Rating calculated from +ve,-ve and Neutral Feedbacks.

If $(\mathrm{k}>=0.0 \& \& \mathrm{k}<=0.2)$

\{

Bench="Not Recommended";

\}

Else if $(\mathrm{k}>0.2 \& \& \mathrm{k}<0.3)$

\{

\}

Bench ="Average";

Else

\section{\{}

Bench ="Most Recommended";

So from the above pseudo code we can find out easily which product is recommended mostly and which one is recommended for average number of users and which one is not recommended by anyone.

\section{IV. . IMPLEMENTATION AND ITS METHODOLOGY}

Implementation is the stage where the theoretical design is converted into programmatically manner. In this stage we will divide the application into a number of modules and then coded for deployment. We have implemented the proposed concept on Java programming language with JEE as the chosen language in order to show the performance this proposed application. The front end of the application takes JSP, HTML AND JAVA BEANS and as a Back-End Data base we took My SQL data base along with a product data set from a local web site. The application is divided mainly into following 2 modules, in which each and 
every module has a set of various sub modules. They are as follows:

1. Admin Module

2. User Module

\section{ADMIN MODULE}

In this module, the admin has to login by using valid user name and password. After login successful he can do some operations such as add categories, add posts, list of all posts, list of all recommended posts, list of all reviewed posts, list of users, list of all search history, update posts, lists of bad reviews by date wise, list of good reviews by date wise, list of all neutral reviews date wise, all friend request, all product reviews and rating results. Here the admin is the one who is maintaining this product data base by inserting a set of items inside the database.

\section{SUB MODULES}

The following are the sub-modules that are available by the admin.Now let us discuss about them in detail as follows:

\section{ADD PRODUCTS POSTS}

In this module, the admin can add the post by including product name, price, description and corresponding product image. Here the products can be shopping, general, stationary or any house holds products. Here the admin maintain a validation steps for developing this sub-module like all the fields should be filled during the insertion of products. If the admin miss any field during the insertion, the product will not be added successfully. Here the validation is written for inserting all fields properly during product insertion and then only the product is added successfully inside the product database.

\section{VIEW ALL POSTS}

In this module, the admin can view the post by searching keyword and can get all the information about the product like product name, price, description and corresponding product image. Here the admin after inserting the products based on category, he can view the set of products that are inserted in the database. All the products are displayed based on category wise and sub-category wise and the user can search the product either with product names or category name or by sub-category wise.

\section{INVENT SENTIMENT ANALYSIS}

The admin can analyze the sentiment based on products from positive sentiment words, products from negative sentiment words, products from neutral sentiment words and View Products Rating based on sentiment words. Here the sentiment analysis is nothing but classifying the different products based on the type of sentiment given by the various users. Actually for each and every product the sentiment /feed back is given by a set of users who try to view the post , based on the keywords they enter in the feedback,the sentiment analysis is done.

\section{USER MODULE}

In this module, there are $\mathrm{n}$ numbers of users are present. User should register before doing some operations. After registration successful he has to login by using authorized user name and password. Login successful he will do some operations like view user details, search 
for products posts, view my search history, view recommended, search for top $\mathrm{N}$ posts and logout.

\section{SUB MODULE: SEARCHES FOR GOOD REVIEWS AND BAD REVIEW}

In this module, user searches for reviews for the post and can get the following information like product name, price, description and corresponding product image. The user can recommend the product and can give review using sentiment words (such as good or bad product like that) based on brand, Quality, Price.

\section{SEARCHES FOR PRODUCTS POSTS}

In this module, user searches for the products or posts after they get login into their account. He will try to enter the search the products based on any field like product name, product category name or product sub category name and so on. Once if the product is matched with any of the field then only the product information is displayed on their login account. If none of the products are matched for the search keyword, then he will get an error like products not matched.

\section{USER RECOMMEND PRODUCTS}

In this module, user searches for the products or posts after they get login into their account. Once after searching any product in his login, he may want to recommend the same for others who registered in the same account. For recommendation he can either recommend individually in his friends list or he can recommend at a time for all friends. Along with recommendation he can also give some valuable comments for the product which will give some more detail information about the product to the end users

\section{USER SEARCH FOR TOP-N POSTS}

In this module, user searches for the products or posts which are almost top rated by the various users.Here if he specify the top $\mathrm{K}$ value from his login, he will get the top rated and accepted products from the database. The top rated will be decided for any product based on the number of positive reviews given by various users for that appropriate product. This function is applied for all the products that are inserted or available in the database, this is not limited for a individual product that is available in the database.

\section{CONCLUSION}

In this paper, we for the first time developed a novel sentiment-based rating prediction method (RPS) to improve prediction accuracy in recommender systems. Initially we developed a social user sentimental measurement approach and calculate each user's sentiment on items/products. Next, we not only consider a user's own sentimental attributes but also take interpersonal sentimental influence into consideration. Later, we take the product reputation factor, which can be inferred by the sentimental distributions of a user set that reflect customer's comprehensive evaluation. Finally, we fuse three factors-user sentiment similarities, interpersonal sentimental influence, and item's reputation similarity into our recommender system to make an accurate rating prediction. As an extension we have also implemented a line chart at the end of the application to show the performance of our proposed application in terms of product features on one axis and number of persons who show interest on products on another axis. By conducting various experiments on our proposed model by taking a sample local website by taking some products into assumption, our simulation results show the sentiment can well characterize user preferences, which help to improve the recommendation performance. 


\section{REFERENCES}

[1] Y. Huang, D. Evans, J. Katz, and L. Malka, "Faster secure two party computation using garbled circuits," in Proc. 20th USENIX Conf. Security Symp., 2011, p. 35.

[2] C. Gentry, "A fully homomorphic encryption scheme," Ph.D. dissertation, Dept. Comput. Sci., Stanford Univ., Stanford, CA, USA, 2009.

[3] Y.-C. Chang and M. Mitzenmacher, "Privacy Preserving Keyword Searches on Remote Encrypted Data," Proc. Third Int'l Conf. Applied Cryptography and Network Security, 2005.

[4] S. Kamara and K. Lauter, "Cryptographic cloud storage," in Proc.14th Int. Conf. Financial CryptographyData Security, 2010, pp. 136-149.

[5] M. Bellare, C. Namprempre, and G. Neven. Security proofs for identity-based identification and signature schemes. J. Cryptology,22(1):1-61, 2009.

[6] W. Sun, S. Yu, W. Lou, Y. T. Hou, and H. Li, "Protecting your right: Attribute-based keyword search with fine-grained owner-enforced search authorization in the cloud," in Proc. IEEE Conf. Comput. Commun., 2014, pp. 226-234.

[7] S. Yu, C. Wang, K. Ren, and W. Lou, "Achieving secure, scalable, and fine-grained data access control in cloud computing," in Proc.IEEE Conf. Comput. Commun., 2010, pp. 1-9.

[8] X. Yang, H. Steck, and Y. Liu, "Circle-based recommendation in online social networks," in Proc. 18th ACM SIGKDD Int. Conf. KDD, New York, NY, USA, Aug. 2012, pp. 1267-1275.

[9] M. Jiang, P. Cui, R. Liu, Q. Yang, F. Wang, W. Zhu, and S. Yang, "Social contextual recommendation," in proc. 21 st ACM Int. CIKM, 2012, pp. 45-54.

[10] X. Qian, H. Feng, G. Zhao, and T. Mei, "Personalized recommendation combining user interest and social circle," IEEE Trans. Knowledge and data engineering. 2014, pp. 1763-1777.

[11] H. Feng, and X. Qian, "Recommendation via user's personality and social contextual," in Proc. 22nd ACM international conference on information \& knowledge management. 2013, pp. 1521-1524.

[12] K.H. L. Tso-Sutter, L. B. Marinho, L. Schmidt-Thieme, "Tag-aware recommender systems by fusion of collaborative filtering algorithms," in Proceedings of the 2008 ACM symposium on Applied computing, 2008, pp. 1995-1999.

[13] F. Li, N. Liu, H. Jin, K. Zhao, Q. Yang, X. Zhu, "Incorporating reviewer and product information for review rating prediction," in Proceedings of the Twenty-Second international joint conference on Artificial Intelligence, 2011, pp. 1820-1825.

[14] X. Ding, B. Liu, and P. S. Yu, "A holistic lexicon-based approach to opinion mining," in 
WSDM '08, pp. 231-240.

[15] Y. Zhang, G. Lai, M. Zhang, Y. Zhang, Y. Liu, S. Ma, "Explicit factor models for explainable recommendation based on phrase-level sentiment analysis," in proceedings of the 37 th international ACM SIGIR conference on Research \& development in information retrieval, 2014.

\section{ABOUT THE AUTHORS}

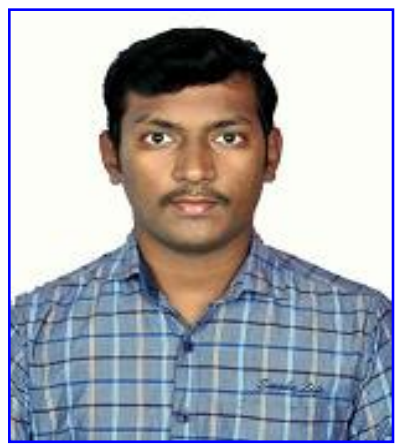

ATHOTA VINAY RAJESH is currently pursuing his 2 Years M. Tech in Department of Computer Science and Systems Engineering (Specialization in Information Technology) at Andhra University College of Engineering, Visakhapatnam, Andhra Pradesh, India. His area of interests includes Data Mining.

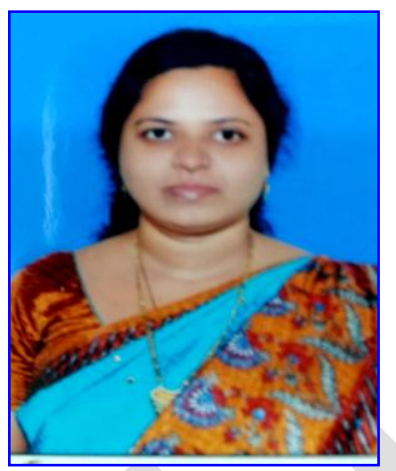

DR. B.PRAJNA is currently working as a Professor in Department of Computer Science and Systems Engineering at Andhra University College of Engineering, Visakhapatnam, Andhra Pradesh, India. She has more than 15 years of teaching experience. Her research interest includes Data Mining. 